\title{
Transesophageal echocardiography (TEE)-induced Mallory- Weiss laceration in a patient who underwent aortic and mitral valve replacement -A case report-
}

\author{
Joo Yeon Lee, Dae Myoung Jeong, Sang Hyun Lee, and Sangmin M. Lee \\ Department of Anesthesiology and Pain Medicine, Samsung Medical Center, Sungkyunkwan University School of Medicine, Seoul, Korea
}

Transesophageal echocardiography (TEE) is a relatively noninvasive and highly valuable diagnostic modality to monitor cardiac surgery. TEE is utilized to estimate the results of the surgical correction or the cardiac function on a real time basis. Accordingly, the frequency of TEE usage is increasing. Previous studies have shown low risk of TEE-associated complications; nonetheless, major gastrointestinal trauma can occur on a rare occasion. We herein present a case of Mallory-Weiss laceration after an intraoperative TEE examination. (Korean J Anesthesiol 2010; 59: S103-S106)

Key Words: Intraoperative, Mallory-Weiss laceration, Transesophageal echocardiography.

Transesophageal echocardiography (TEE) is a relatively noninvasive and highly valuable diagnostic modality to monitor cardiac surgery. TEE is utilized to estimate the results of the surgical correction or the cardiac function on a real time basis with low incidence of TEE-associated complications. Nonetheless, major complications such as gastrointestinal trauma can occur on a rare occasion. Therefore, anesthesiologists using TEE should be aware of potential risks or patient risk factors prone to TEE-induced injuries and manipulate it with caution and safety regulations. In this paper, we present a case of Mallory-Weiss laceration after an intraoperative TEE examination.

\section{Case Report}

A 74-year-old man with infective endocarditis and valvular vegetations was scheduled to undergo aortic and mitral valvular replacement surgery. He had a previous medical history of laparoscopic radical prostatectomy with pelvic lymph node dissection for prostatic cancer, and had received hormonal and radiation therapy with subsequent episodes of rectal bleeding due to radiation-induced prostatitis and telangiectasia. Other than these previous problems, he had no esophageal or gastric related diseases or coagulation abnormalities.

In the operation room, the patient was monitored with

Received: August 27, 2009. Revised: September 24, 2009. Accepted: October 22, 2009.

Corresponding author: Sangmin M. Lee, M.D., Department of Anesthesiology and Pain Medicine, Samsung Medical Center, Sungkyunkwan University School of Medicine, 50, Ilwon-dong, Gangnam-gu, Seoul 135-710, Korea. Tel: 82-2-3410-2470, Fax: 82-2-3410-0361, E-mail: sangminm.lee@samsung.com

(c) This is an open-access article distributed under the terms of the Creative Commons Attribution Non-Commercial License (http:// creativecommons.org/licenses/by-nc/3.0/), which permits unrestricted non-commercial use, distribution, and reproduction in any medium, provided the original work is properly cited. 

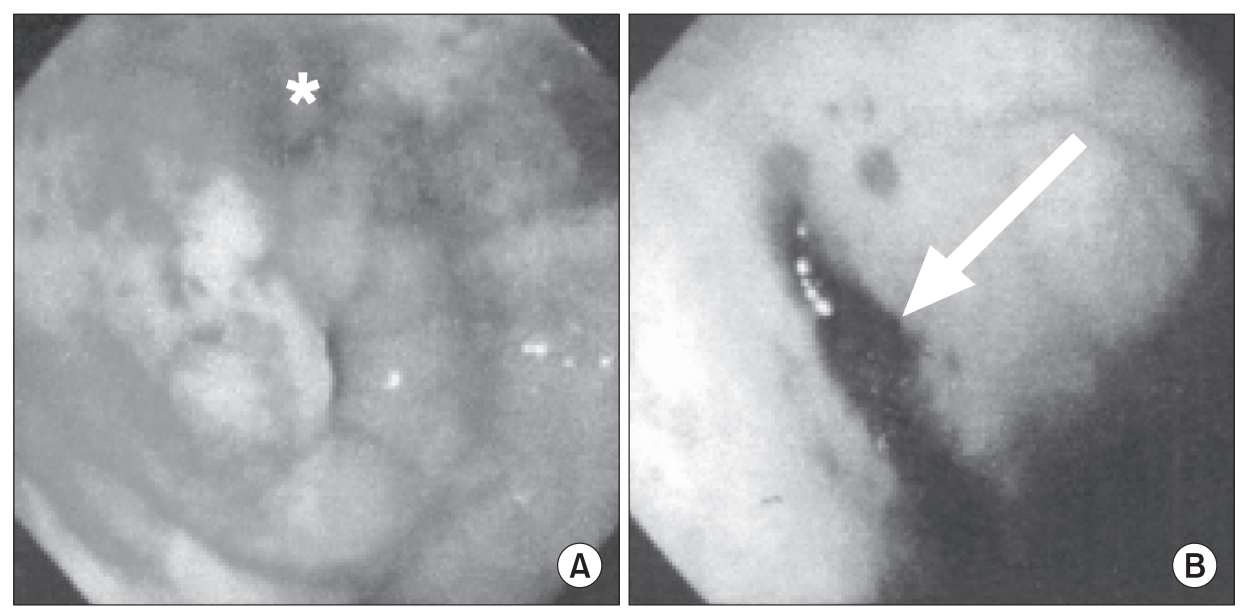

Fig. 1. The lesion $\left(^{*}\right)$ is at the cardia (A). A $3-4 \mathrm{~cm}$ sized deep, linear MalloryWeiss laceration (Arrow) is seen on the esophagogastroduodenoscopic view (B).

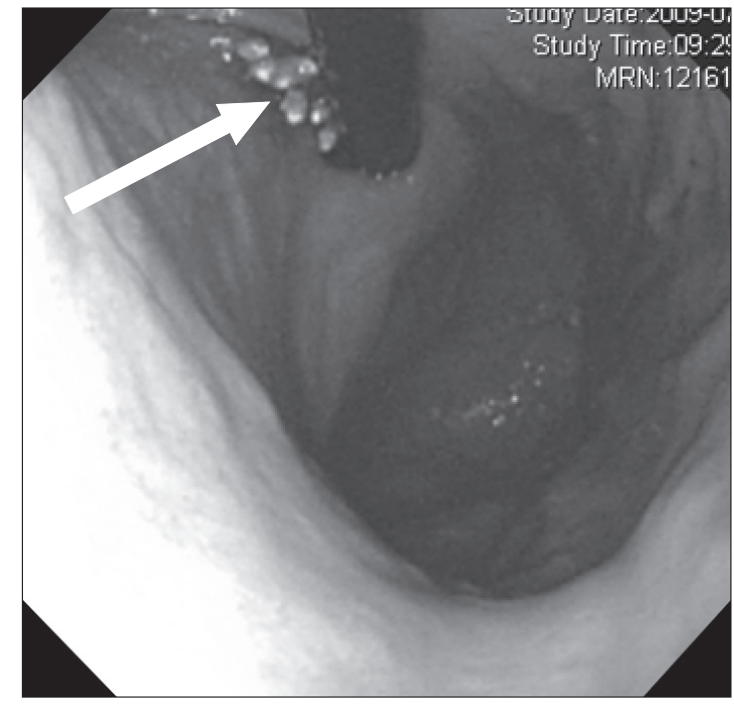

Fig. 2. Homoclips (Arrow) are seen at the cardia and no bleeding is seen on the esophagogastroduodenoscopic view.

electrocardiography, and the continuous arterial blood pressure and noninvasive peripheral oxygen saturation were also assessed. Five minutes after preoxygenation with $50 \%$ to $100 \%$ oxygen, anesthesia was induced with $3 \mathrm{mg}$ of midazolam, 20 $\mathrm{mg}$ of etomidate and $10 \mathrm{mg}$ of vecuronium. The trachea was intubated with a $7.0 \mathrm{~mm}$ endotracheal tube. Anesthesia was maintained with isoflurane at an inspired oxygen fraction of 1.0 and a continuous infusion of $0.8 \mathrm{ug} / \mathrm{kg} / \mathrm{min}$ vecuronium. A central venous cannula was placed at the right internal jugular vein. Thereafter, a transesophageal echocardiography (TEE) probe was inserted into the patient's esophagus through the mouth. The TEE probe was in place throughout the operation.

Before initiating the cardiopulmonary bypass (CPB, the TEE probe was manipulated to visualize both the mitral and aortic valves for both insufficiency and vegetations. During the CPB period, the TEE probe was left in a freeze status.
After weaning the patient off $\mathrm{CPB}$, the TEE was reexamined by a cardiologist to assess the function of the prosthetic aortic and mitral valves. The probe was handled gently while obtaining the appropriate views for the assessment. The mid-esophageal five chamber view, two chamber view and long axis view, the transgastric short axis and long axis view and the deep transgastric long axis view were all visualized. After the operation, the patient was transferred to the CVICU (cardiovascular intensive care unit) without any event.

Four hours after admission to the CVICU, the patient had hematemesis amounting to up to $2 \mathrm{~L}$ of blood. The endotracheal tube was still in place at that time. The Levine tube was irrigated and emergency esophagogastroduodenoscopy (EGD) was performed. The EGD finding was a $3-4 \mathrm{~cm}$ sized deep, linear Mallory-Weiss laceration at the cardia, just below the gastroesophageal junction (Fig. 1). Upon finding the lesion, the bleeder was clipped successfully with no remnant bleeding at the site.

On the second postoperative day, oral intake was started. Eight days after operation, EGD was performed again and there was no trace of bleeding at the lesion (Fig. 2), and was concluded as healing status. The patient was discharged without any further complications related to the Mallory-Weiss laceration.

\section{Discussion}

Intraoperative TEE is widely used because it is relatively noninvasive and safe to apply, and it visualizes the cardiac function and hemodynamic status without interfering with an operation. It supplements transthoracic echocardiography (TTE) before the operation and this modality can estimate the result of the operation on a real time basis. Perioperative TEE examination is recommended in cases of infective endocarditis and doing this is strongly supported by the evidence of 
improved clinical outcomes [1]. However, TEE can sometimes induce direct and indirect oropharyngeal, esophageal and gastric complications. Most of these complications are mild, but sometimes they can be lethal, although this is rare. There are a few reports of injuries outside the alimentary tract (e.g., the spleen) [2].

Generally, TEE is considered to be safe with a low complication rate. Kallmeyer et al. [3] studied 7,200 cardiac surgery patients who underwent intraoperative TEE at a single center. In this study, the TEE-associated complication rate was $0.2 \%$ and the most common complication was oropharyngeal trauma. Odynophagia occurred in $0.1 \%$ of the patients and this accounted for $50 \%$ of all the complications. Gastrointestinal trauma associated with TEE was seen in seven patients $(0.1 \%)$, and two of them had significant upper gastrointestinal (UGI) hemorrhage. Daniel et al. [4] examined 10,419 patients who underwent TEE in a multicenter survey and $0.18 \%$ of the population had TEE-associated complications.

The incidence of TEE-associated direct UGI trauma shows a low risk rate $(0.1-1.2 \%)[4,5]$. However, direct UGI trauma can induce serious complications. In a study by Lennon et al. [5], six of 516 patients who underwent TEE showed major gastrointestinal (GI) complications. Three patients had esophageal and/or gastric tear, two had gastric perforation and one had gastric ulcer. Three of them underwent laparotomy, two received endoscopic treatment and one was treated with transfusion only.

The patient in the present case had a deep, linear laceration at the cardia of the stomach. We supposed that the laceration may have developed while attempting to obtain the deep transgastric long-axis view. The deep transgastric long-axis view is obtained by anteflexion of the TEE probe, and the operator needs to advance or withdraw the probe to achieve more accurate visualization. During this procedure, many operators do not unfold the anteflexed probe, and this may render the esophagus and stomach more susceptible to injury. Thermal and sustained-pressure induced injury can also occur.

In a study by Orihashi et al. [6], the placement of the TEE probe for obtaining the transgastric short-axis view was reviewed in 24 patients. The probe tip was in the stomach in $72.7 \%$ of the patients, at the cardia in $13.6 \%$ and in the esophagus in $13.6 \%$ of the patients. Operators should take caution while searching for the transgastric view, and not just while searching for the deep transgastric view. Also the operators should always be careful not to move the probe unless it is straightened, and not to fold the probe tip for long time so as to avoid a possible thermal or pressure-induced injury.

It is riskier to insert the TEE probe while the patient is anesthetized because the patients can not respond to pain. $\mathrm{CPB}$-applied surgery like the present case has a higher risk of GI trauma because CPB requires full heparinization and it also may induce coagulopathy such as platelet dysfunction, fibrinolysis and coagulation factor consumption.

To decrease the complications, the operators should be careful not to advance the probe more than $30 \mathrm{~cm}$ from the incisor. Experienced operators have fewer complications than do the novices. Daniel et al. [4] reported on 201 cases of failure to insert a TEE probe and $98.5 \%$ of which was due to the lack of operator's experience. Rigid laryngoscope-assisted TEE insertion can also decrease injury to the oropharyngeal mucosal in anesthetized patients [7]. As discussed above, not moving the probe while folded and straightening the probe right after the procedure will lower the complication rate. Maintaining the TEE probe in a freeze status while not in use will help to avoid any thermal injury.

TEE examinations should be avoided or only performed with great caution in patients with significant esophageal or gastric pathology. Esophageal web, stricture or rings, esophageal perforation and obstructive esophageal neoplasm are classified as contraindications for conducting a TEE examination. Cervical spine instability is also a contraindication because adequate neck flexion and extension are needed while inserting the probe. Conditions such as esophageal diverticulum, large hiatal hernia, recent esophageal or gastric surgery, esophageal varices, a history of odynophaiga or dysphagia, cervical arthritis, a history of mediastinal irradiation, oropharyngeal deformities, and severe coagulopathy are also thought to be the relative contraindications [8].

In the present case, the patient had a history of radiation therapy and radiation-induced fragility of the lower intestine. Yet the radiation therapy was not extended to the UGI tract. Therefore, the Mallory-Weiss laceration is not thought to be due to the patient's condition or prior treatment.

Intraoperative TEE is a highly useful modality, especially for reconfirming before surgery the existence of vegetation on heart valves with infective endocarditis, and its usage has become more frequent. Anesthesiologists need to be trained on how to safely manipulate the TEE probe and they should always check the patient's preoperative state for whether the patient has esophagogastric pathologies or coagulopathy. Even if the patient is without pathology, clinicians should always remember that the TEE probe can cause injuries on the GI tract and try to run the probe in a smooth and gentle manner.

\section{References}

1. Thys DM, Abel M, Bollen BA, Cahalan MK, Curling P, Dean RJ, et al. Practice guidelines for perioperative transesophageal echocardiography: a report by the American society of anesthesiologists and the society of cardiovascular anesthesiologists 
task force on transesophageal echocardiography. Anesthesiology 1996; 84: 986-1006.

2. Olenchock SA Jr, Lukaszczyk TT, Reed J 3rd, Theman TE. Splenic injury after intraoperative transesophageal echocardiography. Ann Thorac Surg 2001; 72: 2141-3.

3. Kallmeyer IJ, Collard CD, Fox JA, Body SC, Shernan SK. The safety of intraoperative transesophageal echocardiography: a case series of 7200 cardiac surgical patients. Anesth Analg 2001; 92: 1126-30.

4. Daniel WG, Erbel R, Kasper W, Viisser CA, Engberding R, Sutherland GR, et al. Safety of transesophageal echocardiography. A multicenter survey of 10,419 examinations. Circulation 1991; 83: 817-21.

5. Lennon MJ, Gibbs NM, Weightman WM, Leber J, Ee HC, Yusoff IF. Transesophageal echocardiography-related gastrointestinal complications in cardiac surgical patients. J Cardiothorac Vasc Anesth 2005; 19: 141-5.

6. Orihashi K, Hong Y, Sisto DA, Goldiner PL, Oka Y. The anatomical location of the transesophageal echocardiographic transducer during a short-axis view of the left ventricle. J Cardiothorac Anesth 1990; 4: 726-30.

7. Na S, Kim CS, Kim JY, Cho JS, Kim KJ. Rigid laryngoscopeassisted insertion of transesophageal echocardiography probe reduces oropharyngeal mucosal injury in anesthetized patients. Anesthesiology 2009; 110: 38-40.

8. Savage RM, Aronson S. Comprehensive Textbook of Intraoperative Transesophageal Echocardiography. Philadelphia, Lippincott Williams \& Wilkins. 2004, pp 107-8. 